\title{
Nivel de intensidad de las principales actividades físicas de escolares chilenos: determinación por acelerometría
}

\author{
Intensity level of the main physical \\ activities of Chilean school children: \\ determination by accelerometry
}

\begin{abstract}
The aim of this study was to determine the intensity level of different specific activitiesof chilean school children by accelerometric values. With this objective, 80 children aged 10 to 12 years (39 boys, 41 girls) filled a diary of their activities in a structured format and simultaneously weard an accelerometer for one to three days. The activities of every subject were expressed in counts per minute (cpm). The levels of physical activity (PA). Were classified as sedentary PA / light PA / moderate PA and vigorous PA. Results: 26 different activities were documented. In most of them the difference between boys and girls was weak and no significant. The only vigorous PA was to run. In category "moderate PA" the cpm of plays outdoor was significantly less than those of sports and school gymnastic ( $p$ 0,007)- These results can serve as guiding for establish a scale of values for global evaluation of PA in children schoolers. Key words: school children, physical activities, accelerometer, diary of activities, structured format.
\end{abstract}

\section{INTRODUCCIÓN}

En la lucha contra el sobrepeso y la obesidad de los niños, la cantidad y calidad de su actividad física (AF) y en particular la medición de ella toma cada vez más importancia (1).

Desde varios años se ha difundido la recomendación internacional para que los niños de edad escolar realicen más de 60 minutos diarios de actividad física moderada y vigorosa (AFMV) $(2,4)$. De allí viene la importancia de poder medir lo más exactamente posible el grado de $A F$, tanto a nivel individual como poblacional.

Para esto, la acelerometría parece el instrumento objetivo más confiable, es decir válido y reproducible. Su uso se desarrolló a nivel internacional en muchas áreas de la AF. Un inconveniente, es difícilmente aplicable para el uso clínico, en la práctica de atención primaria, consultorio u otras. Por otra parte, estudios epidemiológicos requieren un número elevado de sujetos, surgiendo un problema de costo. Además, la definición de AFMV depende de su límite inferior en término de "cuentas por minuto" (cpm); este límite es excesivamente variable de una publicación a otra, de 1.000 a 4.000 cpm según la revisión de Guinhouya et al (13) y de 500 a 3.581 cpm según

\section{Claude Godard (1,2) Loreto Carlin(1) Jorge Torres(2), \\ María del Pilar Rodríguez(1) \\ Bárbara Leyton(2) \\ Gabriela Salazar(2)}

1) Escuela de Nutrición y Dietética, Universidad Santo Tomás. Viña del Mar. Chile. 2) Instituto de Nutrición y Tecnología de Alimentos (INTA) Universidad de Chile. Santiago. Chile.

Dirigir correspondencia a: Claude Godard $\begin{aligned} & \text { Claude Godard, } \\ & \text { INTA, Av. El Líbano 5524, Macul, Santiago, Chile. }\end{aligned}$ E mail: claude.godard.m@inta.uchile.cl

Este trabajo fue recibido el 18 de Noviembre de 2015 aceptado con modificaciones el 30 de Diciembre de 2015 y aceptado para ser publicado el 6 de Mayo de 2016.

la revisión de Calahorro et al. (6) (estos valores corresponden al acelerómetro actigraph, actualmente el más usado a nivel mundial). Hace falta una estandarización metodológica en función a la edad del niño y al equipo utilizado.

El cuestionario de AF es un método subjetivo que ha sido ampliamente usado en su aplicación por autoreporte, proxyreporte o por entrevista. Varios estudios de meta-análisis han examinado de manera crítica diferentes cuestionarios publicados en su mayoría en la literatura anglosajona y reportaron mediocres valores de validez, así poniendo en duda su valor y efectividad $(7,9)$. Sin embargo, se siguen utilizando a todas las edades, incluso en preescolares, permitiendo identificar nuevos problemas y futuras direcciones a investigar (10).

Diferentes autores han propuesto determinar tanto el nivel global de AF como el nivel de sus componentes, transformando los datos de la encuestas en MET's (11-13). Tomando el valor "2 MET's" o "3 MET's" u otros límites como punto de corte, se obtiene una clasificación de las AF de niños y una delimitación entre AF leve y AF moderada. Sin embargo, en niños en crecimiento la exacta equivalencia entre la duración de una actividad determinada por encuesta, su intensidad y 
el gasto energético es una incertidumbre.

Otro sistema de clasificación de niveles de AF ha sido propuesto por distribución directa de puntos según una escala pre-establecida. Un cuestionario sencillo de AF con cinco ítems y once preguntas, asociado a un sistema de puntaje con escala de 0 a 10, fue introducido por Burrows et al (14). Este instrumento esta en uso en el Centro de Diagnóstico del Instituto de Nutrición y Tecnología de Alimentos (CEDINTA) de la Universidad de Chile y fue validado en escolares de 8 a 12 años, mostrando una buena concordancia con los valores acelerométricos y con una sensibilidad del 89\% para identificar escolares demasiado sedentarios (15). Sin embargo, la atribución de puntos no tomó en cuenta el nivel de intensidad de los ítems así registrados. En la misma línea, el trabajo de Yañez et al (16) en adolescentes en la Ciudad de Talca, Chile, con un cuestionario de 11 preguntas y puntaje de 11 a 45 deriva a una clasificación de AF en percentiles quizás más objetiva.

El diario de actividad, con su registro prospectivo, fue descrito por Bouchard et al en 1983 (17) y validado por Bratteby et al 1997 (18). Este instrumento ha tenido un uso relativamente reducido en la población pediátrica, por limitada cooperación en el proceso y confiabilidad puesta en duda, aun con adolescentes. Esto ha cambiado, y según estudios recientes, comparando el diario de actividad con acelerometría en niños o adolescentes, la validez de este instrumento fue calificada de razonable y moderada (19-21). Otras publicaciones han mostrado que los diarios de actividad pueden aplicarse en estudios con un número importante de sujetos, más de 300 en el trabajo de Pfitzner et al (22) en Alemania y 400 en el de Pearce et al (23) en Inglaterra. Sin abordar el aspecto de la AF diaria o semanal en su totalidad, la técnica del diario nos parece adecuada para el objetivo de nuestro trabajo, que fue establecer el valor acelerométrico específico de cada AF principal de niños escolares, y el valor de algunas actividades menos habituales. La combinación de datos prospectivos, obtenidos por registro tipo diario y datos acelerométricos respaldará una ponderación más exacta de cada ítem de cualquier cuestionario de AF en niños o adolescentes.

El objetivo del presente estudio fue determinar por valores acelerométricos el nivel de intensidad de diferentes actividades usuales y específicas de niños escolares chilenos.

\section{SUJETOS Y MÉTODOS}

El presente trabajo fue un estudio de tipo descriptivo simple, la muestra determinada por conveniencia y consistió en 93 niños de 10 a 12 años, alumnos de $5^{\circ}$ y $6^{\circ}$ básico. Ellos participaron en forma voluntaria en el proyecto sin ser especialmente seleccionados, en el marco y continuación de la línea de investigación abordada anteriormente (15), y fueron reclutados en tres colegios diferentes de la Región Metropolitana, uno público $(n=30)$, uno privado subvencionado $(n=29)$ y uno particular $(n=34)$. Los niños firmaron junto con sus padres un consentimiento informado y el estudio fue aprobado por el Comité de Ética del INTA. Casos de obesidad o limitación física fueron excluídos del estudio.

Diario: Se utilizó un formato estructurado donde el escolar registró por escrito sus sucesivas ocupaciones del día (figura 1), cada 15 a 30 minutos desde las 13.00 horas (salida del colegio) hasta las 08 horas (entrada al colegio), el tiempo en clases y recreo no figuró en la hoja del diario por estar fijo dentro del programa escolar. No existió selección particular de actividades a escribir por parte de los niños.

Acelerometría: Se utilizó el Actiwatch AW64 (Mini- mitter Co, Bend, Oregón, EEUU) cuyo funcionamiento ha sido descrito en detalle por Puyau et al (24). Este dispositivo miniaturizado $(16,3 \mathrm{~g}, 27 \times 25 \times 9 \mathrm{~mm})$ consiste en un traductor piezoeléctrico y un microprocesador que transforma las aceleraciones omnidireccionales en señales cuantitativas que son registradas en la memoria. La cantidad de minutos con cpm entre 0 y 100 se califica como tiempo en estado de reposo (sedentario) entre 101 y 900 como actividad leve, entre 901 y 2200 como actividad moderada y mayor a 2200 como actividad vigorosa, según la clasificación de Puyau et al (24). El Actiwatch se fija con un cinturón semi - elástico sobre la cadera derecha. Los valores de cpm de cada actividad en particular reportada en el diario y su duración fueron establecidos por análisis y comparación meticulosa con los datos cpm minuto por minuto como lo muestra el ejemplo (figura 1).

Protocolo: El estudio se realizó en los años 2004 y 2005. Las mediciones se efectuaron en los meses de septiembre y octubre. El martes o miércoles de la semana, durante el primer recreo escolar (09 AM) los alumnos recibieron el formulario de diario con instrucciones para su llenado y se les colocó el acelerómetro. Los diarios y la acelerometría se realizaron por un día en 86 sujetos y por tres días en 7 sujetos.

De los 93 participantes, 13 fueron eliminados por abandono (no entrega de diario), diario ilegible o problemas técnicos del acelerómetro.

Los datos de 80 escolares restantes (39 niños, 41 niñas), se analizaron comparando en forma meticulosa cada actividad anotada en sus diarios con los valores de cpm registrados en paralelo, como lo ilustra la figura 1. Los periodos de valores contradictorios fueron descartados.

Estadística: Los datos fueron procesados mediante STATA13 aplicando una estadística descriptiva y comparativa según el caso. Se consideró significativo un $p<0,05$. Se realizó la prueba de Kolmogorov - Smirnov para verificar la normalidad de los datos, y las diferencias fueron determinadas por la prueba no paramétrica de Mann - Whitney.

\section{RESULTADOS}

En los diarios de los 80 niños participantes, se identificaron 26 actividades y ocupaciones; 11 fueron calificadas de principales, escencialmente por la duracción de sus registros, las cuales van de "dormir" a "correr"; sus valores cpm se presentan en figura 2 y tabla 1, (incluyendo el promedio de duración de los registros). Excepto para el valor recreo, la figura separa claramente las 5 ocupaciones sedentarias de las actividades moderadas.

El rubro "furgón y auto" se refiere al transporte en minibús escolar y auto particular para ir al colegio y volver a casa. Estos valores son difíciles de interpretar, pues el acelerómetro registra también los movimientos y sacudidas del vehículo por "eventos" en la calle.

Las actividades catalogadas como "juegos al aire libre" han sido muy variadas desde jugar en un parque público, pasear en bicicleta, andar en monopatín hasta pasear al perro o estar sentado en el pasto conversando con amigos/as. No hubo diferencia significativa entre juegos al aire libre y caminar, pero sí entre juegos al aire libre y deportes o educación física escolar ( $p$ $0,007)$. El ítem "caminar" registro esencialmente los alumnos que van a pie al colegio y vuelven a su casa y también los que han caminado por otro motivo; visita a familiares, compras, entre otros. Su mediana no tuvo diferencia significativa con deportes y educación física escolar.

La actividad "correr" se situó netamente en la categoría de AF vigorosa por su valor mediano superior a 2.200 
cpm. Algunos niños anunciaron haber corrido por periodo relativamente largo (10 a 23 minutos) pero ciertamente con intervalos de reposo y de caminata, lo que explica algunos valores inferiores a $1.500 \mathrm{cpm}$.

Resultados por sexo: La tabla 2 presenta valores de cpm de las actividades principales separadas por sexo y el promedio de duración de los registros. Excepto en deportes (p 0,0081) las diferencias entre niños y niñas fueron leves y no significativas.

Resultados por colegio: En la tabla 3 figuran los valores de cpm de una selección de cinco actividades particulares separadas por colegio. En los alumnos del colegio particular, se obtuvo sólo dos registros de caminar, con certeza porque ellos en su mayoría van al colegio con auto familiar o bus escolar. Deportes programados: Para este item se contó con

\section{FIGURA 1}

Ejemplo de comparación entre grafico de acelerometría y transcripción simplificada del diario por un niño de 12 años.

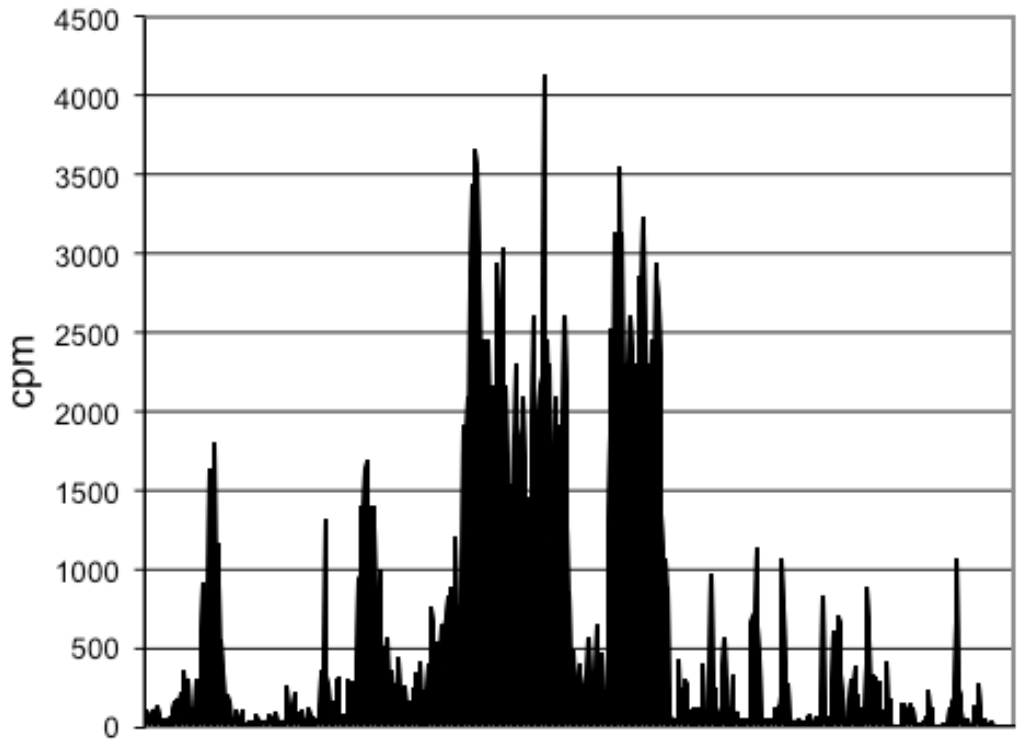

Horas

\begin{tabular}{|c|c|c|}
\hline \multicolumn{2}{|c|}{ REGISTRO DE MIS ACTIVIDADES } \\
\hline $\begin{array}{c}\text { 13.00: salgo del colegio } \\
\text { 13.15: llego a casa, TV } \\
\text { 13.30:almuerzo }\end{array}$ & $\begin{array}{c}17.00 \text { clases de tennis } \\
17.30 \text { regreso a casa en } \\
\text { bicicleta }\end{array}$ & \multirow{2}{*}{$21: 00$ Pilates } \\
\hline $\begin{array}{c}14.00 \text { TV } \\
14.30 \text { voy al football en } \\
\text { bicicleta }\end{array}$ & $\begin{array}{c}18.00 \text { llego a la casa } \\
18.30 \text { veo TV }\end{array}$ & \multirow{2}{*}{$22: 00$ Me duermo } \\
14.45 inicio clases de football & & \\
\hline 15.00 en clases de fooball & $\begin{array}{c}19.00 \text { hago tareas } \\
19.30 \text { once }\end{array}$ & \\
\hline 16.00 descanso & $20: 00$ TV & \\
16.30 inicio clases de tennis & $20: 30$ Estudio & \\
\hline
\end{tabular}


31 registros de diversos deportes, siendo el más registrado el fútbol con 17 registros de 35 minutos en promedio, una mediana de $1.656 \mathrm{cpm}$ y un rango total de 531 a $2.625 \mathrm{cpm}$. Otros deportes registrados en tres y cuatro ocasiones respectivamente: basquetball $n=4,1.366$ a 2.362 cpm; handball $\mathrm{n}=4,1.084$ a $1.906 \mathrm{cpm}$; tenis $\mathrm{n}=3$, de 1.131 a $1.542 \mathrm{cpm}$; voleyball $n=3,359$ a $1.078 \mathrm{cpm}$.

Actividades diversas: A título indicativo, la tabla 4 reporta los valores de cpm de actividades pocas veces reportadas por nuestros escolares.

\section{FIGURA 2}

Valor acelerométrico de las principales actividades estudiadas, clasificadas según las definiciones de Puyau (24).

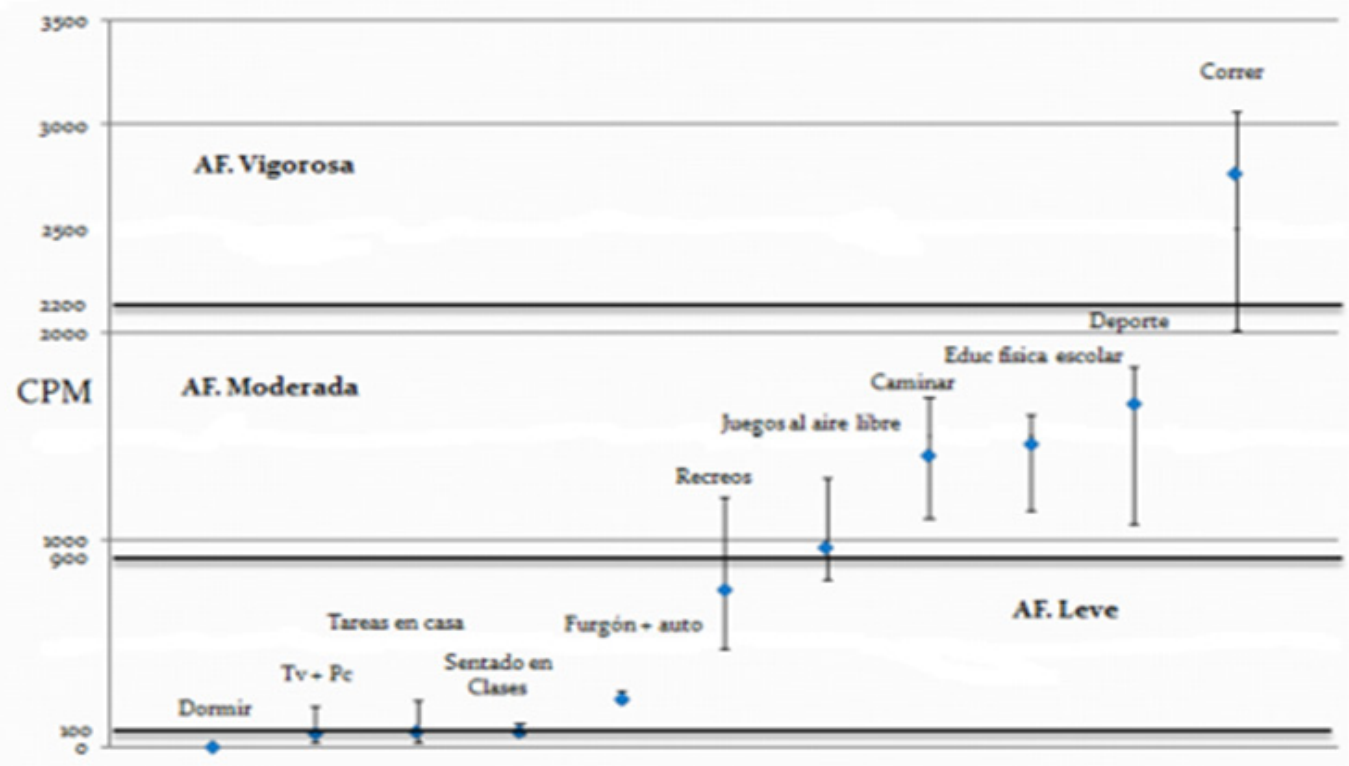

TABLA 1

Cuentas por minuto (percentiles) de 11 actividades estudiadas.

\begin{tabular}{|c|c|c|c|c|c|}
\hline \multirow[t]{2}{*}{ Actividades } & \multirow[t]{2}{*}{$\mathrm{n}$} & \multirow{2}{*}{$\begin{array}{l}\text { Duración } \\
\text { (min) }\end{array}$} & \multicolumn{3}{|c|}{ Cuentas por minuto (cpm) } \\
\hline & & & p50 & p25 & p75 \\
\hline Dormir(1) & 14 & 538 & 7,4 & 4 & 9,5 \\
\hline$T V+P C$ & 48 & 45 & 70 & 44 & 128 \\
\hline Sentado en clase & 58 & 60 & 84 & 58 & 121 \\
\hline Tareas en casa ( 2 ) & 34 & 45 & 85 & 57 & 140 \\
\hline Furgón + auto & 14 & 18 & 239 & 216 & 273 \\
\hline Recreos & 53 & 15 & 766 & 475 & 1206 \\
\hline Juegos al aire libre* & 41 & 30 & 969 & 808 & 1688 \\
\hline Caminar & 42 & 11 & 1412 & 1108 & 1688 \\
\hline Deporte programado * & 31 & 48 & 1656 & 1080 & 1840 \\
\hline Educación Física Escolar & 18 & 80 & 1464 & 1140 & 1601 \\
\hline Correr & 39 & 4 & 2767 & 2007 & 3061 \\
\hline
\end{tabular}




\section{DISCUSIÓN}

La medición de la AF en escolares es necesaria para identificar los problemas actuales de sedentarismo, promover su salud - en particular la salud cardiovascular-y generar políticas publicas que la favorezcan, en la escuela y fuera de ella. Sin embargo, la cuantificacion de la AF habitual es relativamente compleja y al determinarla con métodos mas sencillos y disponibles, la información recolectada debe ser contrastada al mismo tiempo con un método de referencia (25); en nuestro trabajo se utilizó la acelerometría y el diario de actividad informada por el mismo alumno.

El estudio se inscribe en la resurgencia del diario desde algunos años para la evaluación de la AF en niños. Las opiniones divergen a su propósito, entre "no hay datos para recomendarlo" (29) y "recomiendo el uso del diario junto con acelerometría, porque el diario es vital, permite identificar datos a invalidar y falta de observancia de los participantes" (22). La realidad parece intermediaria; si la validación del diario de actividad no fue tema del presente estudio, es un hecho que no ha sido bien establecida por otros. Algunos trabajos calificaron la validez del diario de actividad como moderada o razonable, aportando cifras o valores de correlación con acelerometria que no sobrepasan un $r=0,58(20,21,26,28)$. Sin embargo, en 403 adolescentes portugueses, Machado-Rodrigues et al (19), compararon las cpm con el gasto energético de las actividades registradas por el diario y reportaron una asociación RHO de 0,65 en varones y 0,69 en mujeres. Además, 97\% de los casos entraron en los limites gráficos de Bland-Altman. En este contexto, una mejor confiabilidad de datos de AF por diario podría obtenerse en un futuro con un instrumento recién descrito, que consiste en una pequeña cámara portatil de alta resolución posicionada en el tórax mediante un arnés y que registra todos los movimientos del sujeto (30).

El principal hallazgo de nuestro estudio fue la generación de 26 actividades diferentes, cada una con su valor acelerométrico en cpm. Como lo muestra la figura 2 con las $11 \mathrm{AF}$ principales, su clasificación como sedentaria, leve, moderada y vigorosa ha sido conforme con lo esperado, de acuerdo con la calibración de PUYAU et al (24), tomando en cuenta la amplia distribución de valores individuales de cpm. Esta distribución es similar a la encontrada por Godard et al (27) en niños mas jóvenes 4 a 9 años . La actividad "correr" fue la única catalogada como actividad vigorosa, lo que no puede formar parte de un cuestionario de AF porque se realiza por perio-

TABLA 2

Cuentas por minuto (percentiles) de 11 actividades estudiadas, según sexo.

Niños:

Actividades

n

Dormir

$\mathrm{TV}+\mathrm{PC}$

Sentado en clase

Tareas en casa

Furgón + auto

Recreos

Juegos al aire libre

Caminar

Deporte programado *

Educación física escolar Correr

Niñas:

Actividades

Dormir

$\mathrm{TV}+\mathrm{PC}$

Sentado en clase

Tareas en casa

Furgón + auto

Recreos

Juegos al aire libre

Caminar

Deporte programado *

Educación física escolar

Correr

Duración
(min)
480
45
60
47.5
17.5
15
26.5
13.5
50
80
3

Duración

(min)

550

45

60

32.5

18.5

15

30

9

41

80

6

\begin{tabular}{ccc}
\multicolumn{3}{c}{ Cuentas por minuto (cpm) } \\
p50 & p25 & p75 \\
7.6 & 7.4 & 7.7 \\
74.0 & 44.5 & 163.0 \\
106.0 & 64.8 & 148.0 \\
82.3 & 61.5 & 129.0 \\
248.0 & 214.0 & 300.0 \\
870.0 & 573.0 & 1382.0 \\
928.8 & 720.8 & 1446.0 \\
1321.7 & 1018.5 & 1551.5 \\
1718.0 & 1366.0 & 1840.0 \\
1496.0 & 1195.0 & 1643.0 \\
2767.0 & 2028.0 & 3113.0
\end{tabular}

\begin{tabular}{ccc}
\multicolumn{3}{c}{ Cuentas por minuto (cpm) } \\
p50 & p25 & p75 \\
5.9 & 2.0 & 13.7 \\
88.0 & 43.5 & 109.0 \\
71.0 & 58.0 & 103.0 \\
86.5 & 55.5 & 162.0 \\
238.5 & 220.0 & 258.0 \\
657.0 & 383.0 & 1137.5 \\
1096.0 & 832.0 & 1250.5 \\
1563.0 & 1324.0 & 1764.0 \\
1271.0 & 1064.0 & 1471.0 \\
1515.0 & 1218.5 & 1616.5 \\
2724.0 & 1827.5 & 3061.0
\end{tabular}

* p 0,0081, test Mann-Whitney 
dos generalmente cortos, irregulares, incluso imprevisibles, y difíciles de cuantificar en términos de frecuencia y duración.

La graduación ascendente de las cpm de la figura 2, desde valores bajos, casi sedentarios como ver TV hasta cpm muy altos como "correr" se puede comparar con lo reportado por Bringolf-Isler et al (21) en 189 niños suizos de 6 a 14 años. Estos autores documentaron por acelerometría y diario de actividad un total de 21 actividades diferentes, independientes de la actividad total diaria. Asi, la educación física escolar (gimnasia) y los deportes han sido clasificados como "AF vigorosa", encontrándose en la categoría "AF moderada" en nuestro trabajo. Esta disparidad observada puede explicarse por una diferencia de hábitos entre paises, o por una diferencia en la misma definicion de acelerometría de AF moderada y AF vigorosa $(5,6)$

Llama la atención que nuestros escolares tenian menos AF en la semana cuando jugaban al aire libre que cuando caminaban o practicaban algun deporte. A este propósito, Pearce et al (23), subrrayaron la importancia del tiempo libre después de la escuela, y la oportunidad existente de elevar el nivel de AF moderada si los escolares, - niñas y niñas- no juegan solos, sino que en grupo con amigos (as) o hermanos (as). Se ha co-

TABLA 3

Cuentas por minuto (mediana) de cinco actividades seleccionadas según tipo de colegio.

\begin{tabular}{|c|c|c|c|c|}
\hline Actividades & & Colegio Público & $\begin{array}{l}\text { Colegio particular } \\
\text { subvencionado }\end{array}$ & Colegio privado \\
\hline Recreos & $\begin{array}{c}\mathrm{n} \\
\mathrm{cpm}\end{array}$ & $\begin{array}{c}14 \\
706\end{array}$ & $\begin{array}{c}21 \\
807\end{array}$ & $\begin{array}{c}18 \\
845\end{array}$ \\
\hline Juegos al aire libre & $\begin{array}{c}\mathrm{n} \\
\mathrm{cpm}\end{array}$ & $\begin{array}{c}10 \\
840\end{array}$ & $\begin{array}{c}20 \\
1095\end{array}$ & $\begin{array}{c}11 \\
934\end{array}$ \\
\hline Caminar & $\begin{array}{c}\mathrm{n} \\
\mathrm{cpm}\end{array}$ & $\begin{array}{c}17 \\
1416\end{array}$ & $\begin{array}{c}23 \\
1433\end{array}$ & 2 \\
\hline Deporte programado & $\begin{array}{c}\mathrm{n} \\
\mathrm{cpm}\end{array}$ & $\begin{array}{c}9 \\
1744\end{array}$ & $\begin{array}{c}17 \\
1368\end{array}$ & $\begin{array}{c}5 \\
1078\end{array}$ \\
\hline Educación física escolar & $\begin{array}{c}\mathrm{n} \\
\mathrm{cpm}\end{array}$ & -- & -- & $\begin{array}{c}18 \\
1464\end{array}$ \\
\hline
\end{tabular}

TABLA 4

Valor cpm de 15 actividades menos documentadas.

\begin{tabular}{|c|c|c|c|}
\hline \multirow{2}{*}{$\begin{array}{l}\text { Actividades } \\
\text { (min) }\end{array}$} & $\mathrm{n}$ & \multicolumn{2}{|c|}{ Duración } \\
\hline & & cpm & (rango) \\
\hline Gimnasia en casa ${ }^{1}$ & 10 & 14 & $174-2625$ \\
\hline Volantín & 1 & 25 & 1823 \\
\hline Monopatín & 1 & 15 & 835 \\
\hline Danza & 7 & 23 & $459-1606$ \\
\hline Bicicleta & 6 & 30 & $463-1409$ \\
\hline Pasear al perro & 3 & 22 & $464-906$ \\
\hline Teatro & 3 & 60 & $354-886$ \\
\hline Columpio & 2 & 18 & $467-659$ \\
\hline Dibujar & 4 & 42 & $34-558$ \\
\hline Tareas domesticas & 2 & 18 & $296-598$ \\
\hline Pintura & 2 & 60 & $139-226$ \\
\hline Leer & 6 & 31 & $16-129$ \\
\hline Música² & 6 & 28 & $11-126$ \\
\hline Ajedrez & 2 & 60 & $45-74$ \\
\hline Hablar por teléfono & 2 & 29 & $5-\quad 24$ \\
\hline
\end{tabular}

1 Incluye pilates, argollas, karate, saltar la cuerda.

2 Incluye suma de actividades como tocar flauta, guitarra, piano, cantar. 
mentado el rol del medio ambiente (calles, parques, arboleda) como estimulo a la AF, especialmente cuando caminan desde la casa a la escuela y vuelven a casa (31).

Por otra parte, se observó que los niños del trabajo ya citado (21) eran mas activos que las niñas, tanto a nivel global (diferencia ampliamente reportada a nivel mundial), como en actividades especificas como en el tiempo de recreo en la escuela, la gimnasia escolar, caminar entre otros, y también en temas de sedentarismo. No hemos observado la misma tendencia de género, incluso las niñas de este estudio caminaban un poco más rápidamente que los niños y jugaban mas activamente en parques públicos y plazuelas, diferencias que no fueron significativas. El pequeño número de niñas y niños estudiado, quizás resta importancia a estas diferencias, que no concuerdan con la observación general.

Los datos obtenidos dan una visión nueva sobre la importancia y el valor relativo de algunas actividades principales de la semana, comparándolas entre ellas. Si se quiere evaluar globalmente la AF de un niño en particular por el cálculo de un puntaje con escala de puntos, como lo hemos reportado anteriormente (15), la ponderación del deporte junto a la educación física escolar, caminar y los juegos al aire libre deberá ser diferenciada y no uniforme. Junto con esto, sería recomendable incluir otras actividades tales como ejercicios físicos en casa, tareas domésticas, entre otros.

\section{CONCLUSIÓN}

Este estudio ha proporcionado información sobre la cuantificación de las actividades físicas, libres y en clase, de escolares chilenos reportadas por medición acelerométrica. Se ratifica la importancia del deporte, de caminar y de correr.

\section{RESUMEN}

El propósito del presente trabajo fue determinar por valores acelerométricos el nivel de intensidad de diferentes actividades específicas de niños escolares chilenos. Con este objetivo, 80 niños de 10 a 12 años (39 varones y 41 niñas) llevaron un diario de actividades en un formato estructurado y simultáneamente un acelerómetro por uno a tres días. En cada sujeto las actividades se expresaron en cuentas por minuto (cpm). Los niveles respectivos de actividad física (AF) fueron clasificados en: AF sedentaria/ AF leve / AF moderada / AF vigorosa. Resultados: 26 actividades diferentes fueron documentadas. En la mayoría de ellas la diferencia entre niños y niñas fue leve y no significativa. La única AF vigorosa fue correr. En la categoría "AF moderada" los cpm de los juegos al aire libre fueron significativamente más bajos que las de los deportes y educación física escolar ( $p 0,007)$. Estos resultados pueden servir de guía para establecer una escala de valores en la evaluación global de la AF de niños chilenos.

Palabras clave: Escolares, actividad física, acelerometría, diario de actividad, cuestionario.

\section{BIBLIOGRAFÍA}

1. Government of Chile, Health Ministry... Healthy life program: interventions in untransmitted risk factors. Exempt resolution No 1175 (Nov 2014).

2. Strong WB, Malina RM, Blimkie CJ, Daniel SR, Dishman RK, Gutin $B$ et al Evidence-Based physical activity for school-age youth. J Pediatr. 2005; 146: 732-7.

3. Janssen I. Physical activity guidelines form children and youth. Appl Physiol Nutr Metab. 2007; 32: S109-S121.

4. World Health Organization. Global Recommendations on physical activity for health, WHO Geneva. 2010.
5. Guinhouya BC, Samouda $H$, de Beaufort C. Level of physical activity among children and adolescents in Europe: a review of physical activity assessed objectively by accelerometry. Public Health 2013, 127: 301-11.

6. Calahorro F, Torres-Luque G, Lopez-Fernandezl, SantosLozano A, Garatachea N, Alvarez E. Physical activity and accelerometry: Metodologigal orientations, recommendations and patterns. Nutr Hosp. 2015, 31 (1) 115-22.

7. Chinapaw MJ, Mokkink LB, van Poppel MN, van Mechelen $W$, Terwee CB. Physical activity quesrtionnaires for youth: a systematic review of measurement properties. Sports Med. 2010, 40: 539-63.

8. Farias Junior JC, Lopes Ada S, Florindo AA, Hallal PC. Validity and reliability of self-report instruments for measuring physical activirty in adolescents: a systematic review. Cad Saude Publica 2010, 26: 1669-91.

9. Ekelund U, Tomkinson G, Armstrong N. What proportion of youth are physically active? Measurement issues, levels and recent time trends. Br J Sports Med. 2011, 45 : 859-65.

10. Oliver M, Schofield GM, Kolt GS. Physical activity in prescholers: understanding prevalence and measurement issues. Sports Med. 2007, 37: 1045-70.

11. Hagströmer $M$, Bergman P, De Bourdeaudhuij I, Ortega FB, Ruiz JR, Manios Y et al. Concurrent validity of a modified version of the international physical activity questionnaire (IPAQ-A) in european adolescents: The HELENA Study. Int J Obes. 2008, 32: S42-8.

12. Nava M, Perez A, Herrera H, Hernandez R. Hábitos Alimentarios, Anthropometric-nutritional assessment, dietary habits and physical activity in preschool children, Rev Chil Nutr. 2011, 38:301-12

13. Butte NF. Puyau MR., Adolph AL., Vorha FA:, Zakeri I. Physical activity in nonoverweight and overweight hispanic children and adolescents. Med Sci Sports Exerc. 2007, 9: 1257-66.

14. Burrows R, Diaz E., Sciaraffia V., Gattas V., Montoya A., Lera $L$., Hábitos de ingesta y de actividad física en escolares según tipo de establecimiento al que asisten. Rev Med Chil. 2008; 136:53-63.

15. Godard CM, Rodriguez M, Diaz N, Lera L, Salazar G, Burrows $R$. Value of a clinical test for assessing physical activity in children. Rev Med Chil. 2008, 136: 1155-62.

16. Yañez-Silva A, Hespanol JE, Gomez R, Cossio-Bolaños $M$. Validation of physical activity in school adolescents by questionnaires. Rev Chil Nutr 2014; 41: 360-6.

17. Bouchard C, Tremblay A, Leblanc C, Lortie G, Théricault $G$. A method to assess energy expenditure in children and adults. Am J Clin Nutr 1983, 37: 461-7.

18. Bratteby $L E$, Sandhagen B, Fan H, Samuelson G. A 7-day activity diary for assessment of daily energy expenditure validated by the doubly labelled wáter method in adolescents. Eur J Clin Nutr 1997; 51: 585-91.

19. Machado-Rodrigues AM, Figueiredo AJ, Mota J, Cumming $S P$, Eisenmann JC, Malina RM et al. Concurrent validation of estimated activity energy expenditure using a 3-day diary and accelerometry in adolescents. Scand J Med Sci Sports 2012, 22: 259-.64.

20. Martinez-Gomez D, Wärnberg J, Welk GJ, Sjöström, Veiga $O L$, Marcos A. Validity of the Bouchard acvtivity diary in Spanish adolescents. Public Health Nutr. 2010, 13: 26/-8

21. Bringolf-Isler B, Grize L, Mäder U, Ruch N, Sennhauser FH, Braun-Fahrländer C. Assessment of intensity, prevalence and duration of everyday activities in Swiss school children: a cross-sectional analysis of accelerometer and diary data. 
Int J Behav Nutr Phys Act. 2009, 50: 1-10,

22. Pfitzner R, Gorzelniak L, Heinrich J, Von Berg A, Klümper $C$, Bauer CP et al. Physical activity in German adolescents measured by Accelerometry and activity diary: Introducing a comprehensive approach for data management and preliminary results. PLoS One 2013; 8(6); e65192.

23. Pearce M, Page AS, Griffin TP, Cooper AR.Who children spend time with after school: associations with objectively recorded indoor and outdoor physical activity. Int J Behav Nutr Phys Act. 2014, 11(1): 45.

24. Puyau MR Adolph AL, Vohra FA et al. Validation and calibration of physical activity monitors in children, Obes Res. 2002; 10: $150-7$.

25. Trost, SG. Mciver, KL., Pate, RR. Conducting accelerometer based activity assessments in field-based research. Med SCi Sports Exer.c 2005. 37 (11) S531-43.

26. Wen LM, Van der Ploeg HP, Kite J, Cashmore A, Rissel C. A validation study of assessing physical activity and sedentary behavior in children aged 3 to 5 years. Pediatr Exerc Sci.
2010; 22(3): 408-20.

27. Godard, CM, Román, M. Rodriguez MP. Leyton, B. Salazar $G$. Variability of physical activity in 4-10-year-old children: A study by accelerometry. Arch Argent Pediatr. 2012; 110 (5) : 388-93.

28. Floro JN, Dunton GE, Delfino RJ. Assessing physical activity in children with asthma: convergent validity between accelerometer and electronic diary data. Res Q Excerc Sport 2009. 80(2): 153-63.

29. Bradley J, O'Neil B, Kent L, Hulzebos EH, Arets B, Hebestreit $H$. Physical activity assessment in cystic fibrosis: $A$ position Statement. J Cyst Fibros 2015, 14(6): e25-32.

30. Kelly P, Thomas E, Doherty A, Harms T, Burke Ó, Gershuny J, Foster C. Developing a Method to test Validity of 24 Hour Time Use Diaries Using Wearable Cameras: A Feasibility Pilot. PLoS One 2015 3;10(12) e0142198.

31. Armstrong N, Welsman JR. The physical ctivity patterns of European youth with reference to methods of assessment. Sports Med. 2006; 36(12): 1067-86. 